\title{
Argumentation prompts mediating student resource use on conceptual problems
}

\author{
Carina M. Rebello \\ Department of Physics and Astronomy, Purdue University, 525 Northwestern Ave., West Lafayette, IN, USA/47907
}

\begin{abstract}
Scientific argumentation has been highlighted in the Next Generation Science Standards (NGSS) as one of the key science and engineering practices. While there have been relatively few studies on student argumentation in undergraduate physics, research has shown that the use of argumentation prompts can potentially improve not just the argumentation quality, but also the conceptual quality of student responses to written conceptual physics problems. In this study we extend previous research to look more closely at the impact of construct and evaluate argumentation prompts on the resources students bring to bear on conceptual physics problems. We examine the kinds of conceptual resources used by future elementary teachers when justifying their claim and also when they constructed a rebuttal to a counterargument.
\end{abstract}

\section{INTRODUCTION}

Argumentation has been highlighted as a key scientific practice in recent NGSS [1] and plays a crucial role in scientific inquiry [2]. Engagement in argumentation can facilitate an arguing-to-learn process in which students construct scientific understandings through justifying, evaluating, and confronting varying scientific views [2]. Prior research has shown that those who write higher structural quality of arguments also tend to have a higher conceptual quality of content knowledge [3,4]. Yet, research has shown that students have difficulty constructing arguments and producing counterarguments or rebuttals without appropriate scaffolds or question prompts $[5,6]$. Facilitating scientific argumentation with the inclusion of scaffolds for construction or evaluation of arguments can improve quality of argument structure and problem-solving performance on conceptual problems [2].

The study is important for three reasons. First, although argumentation has been widely researched in literature, there has been relatively little research on argumentation in the context of physics. Second, there has been little research on the kinds of resources students bring to bear when constructing or evaluating arguments. Third, given the importance of scientific argumentation in NGSS as one of the eight science and engineering practices, it is imperative that pre-service teachers of science become aware and adept at constructing and evaluating arguments. These experiences with argumentation are important especially for future elementary teachers, who often take only a single physics course.
In light of these reasons, this study explores how future elementary teachers incorporate formal, experiential and/or pedagogical resources into their written arguments. Specifically, this study investigates whether argumentation prompts can enhance the number of resources to justify their reasoning and if those resources are used productively. The research questions that guided this study are: (a) What kinds of resources do future elementary teachers incorporate in their arguments? (b) What additional resources, if any, do they use in their rebuttal statements that they did not use in their claim and justification? (c) To what extent are these resources applied in productive, scientifically appropriate ways?

\section{THEORETICAL FRAMEWORK}

\section{A. Resources}

Resources are pieces of knowledge that a student uses as part of their reasoning process [7]. A resource by itself is neither correct nor incorrect, rather it can be used productively or unproductively to respond to a question in a given context. Novice learners are not necessarily consistent in their use of resources while describing or explaining the same underlying physical phenomenon across different contexts.

\section{B. Argumentation}

Kuhn [8] identified five argumentation skills: generating causal theories, offering supporting evidence, envisioning conditions that undermine one's theory, generating 
alternative theories, and rebutting alternative theories. Thus, successful argumentation requires a problem solver to develop and articulate a reasonable solution, support the solution with data and evidence, as well as identify alternative solutions, and develops theories to rebuttal alternative solutions [9]. Argumentation can be divided into two types of activities, constructing an argument and evaluating an argument. In this study, argumentation is viewed as consisting of two intertwined dimensions content-based arguments or incorporation of content knowledge and argumentative discourse [10]. Attending to both these dimensions ensures that students are arguing-tolearn. Learning science involves understanding, constructing, and evaluating varying arguments. Also deep engagement with science content requires that students constantly use scientific concepts and principles to construct written arguments [10].

\section{METHODS}

\section{A. Participants and Data Collection}

Participants in this study were 168 future elementary teachers enrolled in a conceptual physics for elementary teachers course at a large U.S. Midwestern university. Over $90 \%$ of the students were female and a majority had sophomore standing or higher. A majority of the students had not taken high school physics and had no prior training with argumentation. The course goal was not only to facilitate learning of physics content but also help preservice teachers develop pedagogical content knowledge relevant to teaching physics topic at the elementary grade level.

A week prior to this study, all students received a 40minute training phase describing criteria for a good argument. Procedure of this training was similar to that used by Nussbaum and Schraw [11]. Students were then assigned to one of two conditions depending upon where they were seated: evaluate and construct. Each of these conditions corresponded to the type of argumentation task they would receive. The argumentation tasks were provided to each condition three weeks into the semester. The data were collected during the lecture in class. Students were allowed to discuss in groups of about 3-4 students for about 20 minutes and were then provided an additional 20 minutes to write their own argument.

The argumentation tasks are similar to those employed in Physics by Inquiry ( $P b I)$ [12] in that, $P b I$ utilizes both open-ended question tasks (construct a response) and hypothetical student debate tasks requiring application of conceptual knowledge and reasoning skills (evaluate and form a response). In addition to the argumentation task, students were provided relevant question prompts to facilitate meaningful scientific written argumentation [2]. Specifically, prompts facilitating (a) argument construction (b) evaluation of arguments posed by fictitious people in a hypothetical debate. Construct prompts were adapted from Mason and Scirica [13]. Evaluate prompts were adapted from Jonassen et al. [14]. Figs. 1 and 2 show the construct and evaluate tasks used to collect data in this study.

As a student teacher you are asked to accompany your elementary class on a
school trip in a van. A kid notices a sign about a passenger side airbag and asks.
"Why do cars have airbags?"
What is your answer? Construct an argument to justify your answer. Remember to
consider:
- What reasons and evidence support your answer?
- What is a reasonable alternative answer that an elementary kid may provide?
- How would you help the elementary kid understand that their position is
incorrect, and your position is correct?

\section{FIG 1. Construct argument task.}

As a student teacher you are asked to accompany your elementary class on a
school trip in a van. A kid notices a sign about a passenger side airbag and asks.
"Why do cars have airbags?" Two other kids jump in to respond.
Amy: "With an airbag you don't go as fast, so if you hit the front in a crash you
won't get as hurt."
Ann: "No, that's not true. With an airbag you still go the same speed, but you
don't slow down as much, you keep going."
Which answer above do you agree with? Or do you have another answer? Explain
and justify your answer. Remember to consider:
- What reasons and evidence support your answer?
- What is a reasonable alternative answer that an elementary kid may provide?
- How would you help the elementary kid understand that their position is
incorrect, and your position is correct?

FIG 2. Evaluate argument task.

\section{B. Analysis}

A total of 168 written responses (87 evaluate condition and 79 construct condition) were collected. All responses were openly coded by two-independent raters for resources students utilized in their arguments. After independent coding, the raters discussed all codes to reach $100 \%$ agreement. Codes were then collapsed to reduce redundancies by combining similar codes [15].

Additionally, two-independent raters coded for conceptual quality of students' written argument, following similar coding procedures. Arguments may consist of multiple justifications or resources to provide a rationale to support an assertion or claim. To code for scientific conceptual quality within the claim and rational and for the overall written argument, Zohar and Nemet's [16] framework on content of the justification in an argument was adapted. Their framework evaluates what scientific knowledge students incorporate in their argument by 
examining the quality of each justification embedded with in. In this framework, strong arguments should consist of multiple justifications that incorporate relevant and scientifically appropriate concepts to support a claim [17]. In contrast to examining the quality of each justification or how a resource was applied, the overall argument and claim and rationale statements was holistically evaluated. The coding scheme is as follows: "Justified" - having scientifically correct, well justified argument; "Adequate" having scientifically incorrect or partially correct and could be incomplete justification; and "Problematic" - having scientifically incorrect or partially incorrect, not well or non-relevant justifications. Each student response was coded twice: First considering only the claim and reasoning provided, and second holistically with the rebuttal included. The intent was to investigate whether additional resources, if any, provided in the rebuttal were used productively.

\section{RESULTS}

A brief description of common resources used is in Table 1. Resources both conditions are in Figs. 3 and 4.

TABLE I. Resources on tasks in Figs. 1 and 2

\begin{tabular}{ll}
\hline \hline Resource & \multicolumn{1}{c}{ Brief Description } \\
\hline Time Increase & Air bags increase time of collision \\
\hline Equation & Force = Momentum Change / Time \\
\hline $\begin{array}{l}\text { Divide by a } \\
\text { Larger Number }\end{array}$ & $\begin{array}{l}\text { In the equation above, you divide by } \\
\text { a larger number, so force decreases }\end{array}$ \\
\hline Slow Down & Air bags causes you to slow down \\
\hline Force Decreases & Air bags decrease the force on you \\
\hline Material Nature & Refers to softness of the airbag \\
\hline Makes you Stop & Airbags make you stop moving \\
\hline Same Speed & Same speed (momentum) change \\
(Momentum) & with or without airbags. \\
\hline $\begin{array}{l}\text { Force (Not) } \\
\text { Transferred }\end{array}$ & $\begin{array}{l}\text { You do (not) transfer force to } \\
\text { airbag. }\end{array}$ \\
\hline Barrier & Airbags are a barrier to your motion \\
\hline Do Experiments & $\begin{array}{l}\text { Children can do (see) an experiment } \\
\text { (Show Videos) }\end{array}$ \\
\hline \hline
\end{tabular}

The following common general trends can be observed for both conditions. First, the many of the most prevalent resources (such as 'time increases', 'equation', and 'divide by larger' ) are used by students almost equally commonly in both the evaluate and construct conditions.

These most prevalent resources, are mostly invoked in the 'claim and reason' stage of the argumentative response, but less so in the 'rebuttal' stage of the response. This indicates that when students make a claim and provide supporting reasons, they tend to rely on resources that are most likely acquired through formal knowledge learned in the class i.e. through the lecture. Second, in both conditions students seem to use a wider range of resources in the 'rebuttal' stage of their argumentative response than in the 'claim and reasoning' stage of their response.

The additional resources utilized by students in their rebuttal stage are sometimes based on the counter position that they present (not shown in Figs). For instance students invoke the 'material nature' of the air bag in the rebuttal stage. Upon further examination, we find that most of the students who used the 'material nature' resource often invoked this resource in response to the counter position.

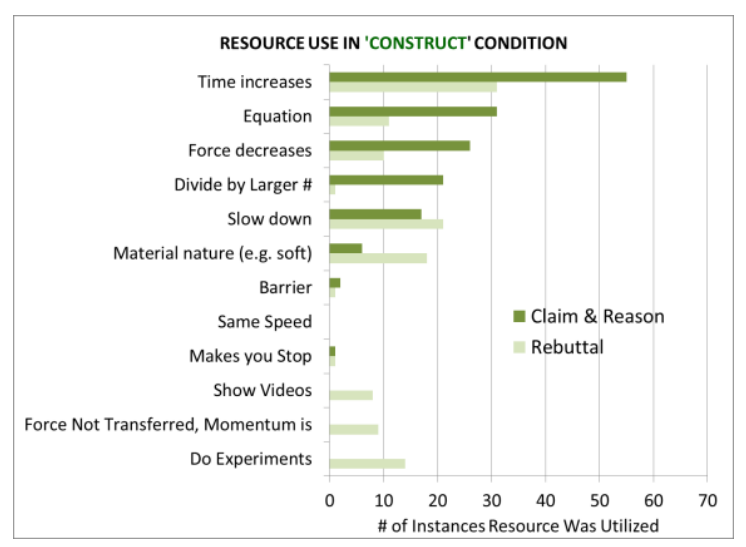

FIG 3. Resources used by the construct condition.

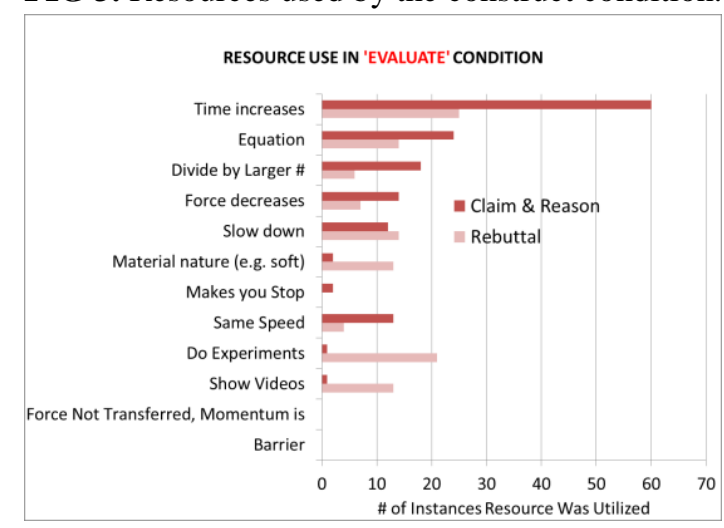

FIG 4. Resources used by the evaluate condition.

Additionally, in the rebuttal stage students often tended to utilize resources based on the instructional strategies used in class including hands on experiments, demos and videos. Students had used and discussed this video in the lab and it had also been discussed in the lecture. Therefore, when students had to design a rebuttal for an elementary child's counter position, they used experiential rather than formulaic scientific reasoning as part of their resource kit. 
We also see some differences between resources used in the two conditions, especially in the 'rebuttal' stage of the argumentative response. For instance, videos and experiments were more prevalent in the 'evaluate' while 'force is not transferred' was more prevalent in the 'construct' condition. We believe that a reason for this difference is that the 'evaluate' condition relied on counter positions from the hypothetical responses provided in the question, while the 'construct' condition tended to create hypothetical counter positions based on elementary children's alternative conceptions discussed in class.

We were also interested in finding out the extent to which students applied their resources in productive and scientifically appropriate ways. To do so, we coded each response twice. First, only the claim and reasoning (i.e. response to "What reasons and evidence support your answer?") were coded as "justified", "adequate" or "problematic". Second, the entire response, including the rebuttal (i.e. response to "How would you help the elementary kid understand that their position is incorrect, and your position is correct?") was coded using the same scheme. The intent was to measure whether the additional resources that were invoked by the students in their rebuttals were being used appropriately. We found that approximately $30 \%$ of the responses changed in the quality of their justification provided. Unfortunately, we also found that the justification quality of a vast majority of these responses (about $20-25 \%$ out of $30 \%$ ) declined with the use of additional resources. This means that although students were using additional resources while writing their rebuttals, they were not necessarily doing so appropriately. This trend was similar across both conditions. Future work will expand this investigation to other topics and other audiences.

[1] NGSS Lead States, Next Generation Science Standards: (Natl Acad Press, Washington, DC, 2013)

[2] R. Driver, et. al, Sci. Educ. 84, 287-312 (2000)

[3] C. M. Rebello, E. Sayre, N. S. Rebello, in Proceedings of the 10th International Conference of the Learning Sciences, edited by V. Aalst, B. J. Reiser, C. H. Silver, \& K. Thompson (Sydney, Australia, 2012)

[4] D. Clark, V. Sampson, JRST, 45, 293-321 (2008)

[5] A. Reznitskya, et. al., Discourse Process, 32 (2-3), 155-175 (2001)

[6] X. Ge and S. M. Land, ETR\&D. 52, 1042-1629 (2004).

[7] D. Hammer, Am. J. Phys. 68, S52-S59 (2000)

[8] D. Khun, The skills of argument, (University Press, Cambridge, UK, 1991)

\section{CONCLUSIONS}

Prior research has shown connections between argumentation quality and scientific conceptual quality of students' responses. Yet, argumentation as process for learning is seldom emphasized in physics classrooms [18].

We find that use of argumentative prompts can help students activate a wider repertoire of reasoning resources than merely asking them to provide an answer and explain their reasoning. Prompts that ask students to consider counter positions and rebuttals to these counter positions can be a useful tool to facilitating students to activate a wider range of resources. For a about a quarter of these students, the task of constructing these rebuttals to hypothetical counter positions, results in the inappropriate use of these additional resources.

The main limitation of this study is that it is confined to a single task in a single class for future elementary teachers. In spite of this limitation, there are two important broader impacts of this research. First, the study demonstrates that the use of argumentative prompts can be a useful tool to facilitate students to bring to bear previously inert experiential knowledge in the form of new resources and ways of thinking about the problem. This shift in framing can be especially useful for future teachers who need to develop appropriate epistemological framing to facilitate future students in their learning of science. Second, the study demonstrates, that argumentative prompts may also have value as a diagnostic tool in that they in many cases reveal that students may not necessarily use resources in scientifically appropriate ways. Therefore this study demonstrates that the use of argumentative prompts may reveal alternative student conceptions about a topic.

[9] K. L. Cho, D. H. Jonassen, ETR\&D, 50, 5-22 (2002)

[10] H. Jin et. al. JRST, 52, 1132-1162 (2015).

[11] E. M. Nussbaum, G. Schraw, JEE, 76, 59-92 (2007)

[12] L. C. McDermott, Physics by Inquiry (Wiley, NY, 1996).

[13] L. Mason, F. Scirica, Learn. Instr. 16, $492-509$ (2006).

[14] D. H. Jonassen, et. al, JEE, 98, 235-254 (2009).

[15] J. W. Creswell, Qualitative Inquiry and Research Design: Choosing among Five Approaches, 2nd ed. (Sage Publishing, Thousand Oaks, CA, 2007).

[16] A. Zohar, F. Nemet, JRST, 39, 35-62 (2002)

[17] V. Sampson, D. B. Clark, Sci. Ed., 92, 447-472 (2008)

[18] J. Osborne, Science, 328, 463-466 (2010) 